\title{
CISTECTOMÍA PARCIAL LAPAROSCÓPICA: NUESTRA SERIE
}

\author{
Octavio A. Castillo',2, Juan C. Aranguibel', Rafael Sánchez-Salas?', Alejandro Foneron', \\ Gonzalo Vitagliano', Manuel Díaz' y Marcelo Fajardo?.
}

'Sección de Endourología y Laparoscopia Urológica, Clínica Santa María. Chile.

${ }^{2}$ Departamento de Urología, Facultad de Medicina, Universidad de Chile. Chile.

\begin{abstract}
Resumen.- OBJETIVO: La cistectomía parcial es un procedimiento establecido de preservación vesical. En el campo de la oncología, la selección del paciente es fundamental para obtener resultados óptimos. Presentamos nuestra experiencia inicial de cistectomía parcial laparoscópica en un grupo seleccionado de pacientes portadores de diferentes patologías vesicales tanto benignas como malignas.
\end{abstract}

MÉTODOS: Entre julio del 2002 y septiembre del 2006, 9 pacientes fueron sometidos a una cistectomía parcial laparoscópica en nuestra institución. Las patologías que indicaron la cirugía fueron; presencia de cuerpo extraño intravesical, endometriosis, adenocarcinoma del uraco, carcinoma vesical de células transicionales, lesiones adquiridas del uraco y un tumor extravesical. Se analizaron la variables de tiempo quirúrgico, tasa de conversión, sangrado, días de permanencia de sonda vesical y estadía hospitalaria.

RESULTADOS: Todos los pacientes fueron operados en su totalidad por vía laparoscópica transperitoneal sin registrarse conversiones a cirugía abierta. El tiempo operatorio promedio fue de 77.7 min (rango 30-120 de minutos). Ningún paciente requirió transfusión sanguínea. No se presentaron complicaciones intraoperatorias. La estadía promedio de hospitalización fue de 4 días (rango 3-5 días), con un tiempo promedio de permanencia de sonda vesical de 7 días (rango 5-10 días).

CONCLUSIONES: La cistectomía parcial laparoscópica es un procedimiento quirúrgico realizable y aplicable para patología tanto benigna como maligna. En los tumores de células transicionales y carcinomas del uraco el resultado y seguridad oncológica deben prevalecer y esto se logra con la selección adecuada de pacientes.

Palabras clave: Cistectomía parcial. Laparoscopia.

Summary.- OBJECTIVES: Partial cystectomy is a recognized bladder sparing surgical technique. Patient selection is essential in order to optimize long term results. We present our initial experience with laparoscopic partial cystectomy performed in a selected group of patients that includes both benign and malignant lesions.

METHODS: Nine patients were surgically treated with laparoscopic partial cystectomy between July 2002 and September 2006 in our institution. Partial cystectomy 
was indicated for the treatment of a foreign body, endometriosis, urachal adenocarcinoma, trancisional cell carcinoma, acquired lesions of urachus and extravesical tumor. Analyzed surgical variables were operative time, conversion rate, blood loss, hospital stay and catheterization period.

RESULTS: Nine patients with bladder lesions were laparoscopically operated for partial cystectomy. Transperitoneal technique was employed and no conversion to open surgery was performed. Median operative time was $77.77 \mathrm{~min}$ (range $30-120 \mathrm{~min}$.). No patient received any transfusion. No intraoperative lesions were verified. Median hospital stay was 4.22 days (range 3$5 \mathrm{~d}$.), median catheterization period was 7 days (range 5-10d.).

CONCLUSIONS: Laparoscopic partial cystectomy is a doable surgical procedure. It is a useful technique for both benign and malignant bladder disease. In TCC and urachal carcinoma oncological safety prevails. $\mathrm{Pa}-$ tient selection is essential for this procedure.

Keywords: Partial cistectomy. Laparoscopy.

\section{INTRODUCCIÓN}

En los últimos años, las mejoras en las habilidades laparoscópicas de los urólogos y el advenimiento de instrumentos para facilitar las técnicas de sutura intracorpórea han creado interés renovado en la cirugía reconstructiva laparoscópica del tracto urinario inferior. De este modo, los procedimientos de resección de lesiones vesicales tienden cada vez más a ser menos invasivos encontrando en la laparoscopia una nueva arma terapéutica (1). Por otro lado, la cistectomía parcial encuentra sus principales indicaciones en las lesiones, tanto malignas como benignas, con localización a nivel de la cúpula vesical y paredes laterales vesicales, lo que permite la realización del procedimiento sin afectar de forma determinante la función vesical (2).

Presentamos aquí nuestra experiencia inicial de cistectomía parcial laparoscópica en un grupo seleccionado de pacientes portadores de diferentes patologías tanto benignas como malignas.

\section{MATERIAL Y MÉTODOS}

En el período comprendido entre julio del 2002 y septiembre del 2006, diez pacientes fueron sometidos a una cistectomía parcial laparoscópica en nuestra institución. En los casos de lesiones tumorales malignas, se explicó de manera clara a los pacientes los beneficios y riesgos presentes en la realización del procedimiento. Se indicó la realización del procedimiento a pacientes con los siguientes diagnósticos: cuerpo extraño intravesical, endometriosis, adenocarcinoma del uraco, carcinoma vesical de células transicionales, lesiones adquiridas del uraco y un tumor extravesical (Tabla I).

No hubo criterios de exclusión presentes debido al volumen o características de las lesiones. Todos los pacientes se sometieron a una cistoscopia y una tomografía axial computada preoperatoria.

La información fue recolectada prospectivamente en una base de datos y se analizó en forma retrospectiva. Las variables analizadas para el estudio fueron: tiempo operatorio, estadía hospitalaria, sangrado intraoperatorio, tasa de conversión quirúrgica, tiempo de sonda y complicaciones perioperatorias.

En los pacientes con lesiones de carcinoma invasivo de células transicionales, se realizó linfadenectomía pélvica de manera habitual como lo hacemos en la cistectomía radical.

\section{TABLA I. DIAGNÓSTICOS PREOPERATORIOS EN PACIENTES SOMETIDOS A CISTECTOMÍA PARCIAL LAPAROSCÓPICA.}

\begin{tabular}{|lc|}
\hline DIAGNÓSTICO & $\mathbf{N}$ ○ \\
\hline Cuerpo extraño intravesical & 1 \\
\hline Tumores extravesicales & 1 \\
\hline Adenocarcinoma del uraco & 1 \\
\hline Carcinoma de células transicionales & 1 \\
\hline Lesiones congénitas del uraco & 3 \\
\hline Endometrioma intravesical & 3 \\
\hline
\end{tabular}




\section{TÉCNICA QUIRÚRGICA}

Todos los pacientes recibieron profilaxis antibiótica preoperatoria con una cefalosporina de $2^{a}$ generación. Se coloca al paciente en decúbito dorsal, en posición de litotomía (Figura 1). El cirujano se ubica en el lado izquierdo del paciente y el asistente frente a él. Un segundo asistente debe colocarse entre los miembros inferiores del paciente para realizar la cistoscopia simultánea durante el acto operatorio. De esta forma, el cirujano obtiene al mismo tiempo las visiones laparoscópica y endoscópica. Se crea el neumoperitoneo por técnica de Veress y se mantiene presión intraabdominal con $\mathrm{CO} 2$ a $15 \mathrm{mmHg}$. Se utiliza una técnica de tres portales (Figura 2), con el trocar inicial de $10 \mathrm{~mm}$ ubicado a nivel supraumbilical para la introducción del laparoscopio de 0 grados, y dos trócares de $10 \mathrm{~mm}$ de ubicación paraumbilical - pararectal para los lados derecho e izquierdo, respectivamente. Con el uso del doble campo visual, que permite verificar el margen de resección, se procede a realizar la resección de la pared vesical comprometida mediante el Bisturí Armónico ${ }^{\circledR}$ (Ethicon, Endosurgery).

La cistoscopia se realiza con bajo volumen de solución, y una vez que se incide la vejiga, se omite el uso de solución ya que el gas ocupa el lumen vesical. Se procede a la extracción del espécimen operatorio con el uso del Endobag® (Ethicon, Endosurgery) y una vez realizado lo anterior, reparamos el defecto vesical mediante técnica de sutura intracorpórea en un plano, con sutura 3 -0 de ácido poliglicólico multifilamento.

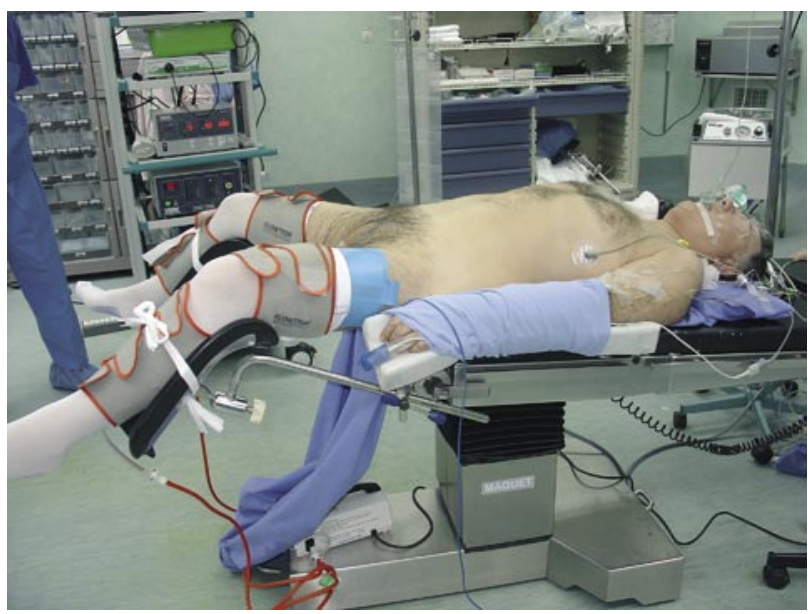

FIGURA 1. Posición de litotomía para la realización de la cistectomía parcial.

\section{RESULTADOS}

Todos los pacientes de la serie fueron operados en su totalidad por vía laparoscópica transperitoneal, sin registrarse conversiones a cirugía abierta. El tiempo operatorio promedio fue de $77.7 \mathrm{~min}$ (rango 30-120 minutos). El sangrado intraoperatorio fue insignificante y ningún paciente requirió tranfusión sanguínea. La estadía promedio de hospitalización fue de 4 días (rango 3-5 días), con un tiempo de permanencia de sonda vesical promedio de 7 días (rango 5-10 días). No se presentaron complicaciones intraoperatorias, postoperatorias ni mortalidad.

El informe anatomopatológico de las piezas quirúrgicas reportó: tres quistes del uraco, tres endometriomas vesicales, un carcinoma de células transicionales, un adenocarcinoma del uraco y un leiomioma vesical. Todos de los márgenes de resección quirúrgica fueron negativos. El tiempo promedio de seguimiento fue de 24 meses (rango 6 - 36 meses), sin registrarse recurrencias en los pacientes tratados por patología tumoral maligna o benigna. De igual forma no se ha constatado compromiso de la funcional vesical tras la realización del procedimiento.

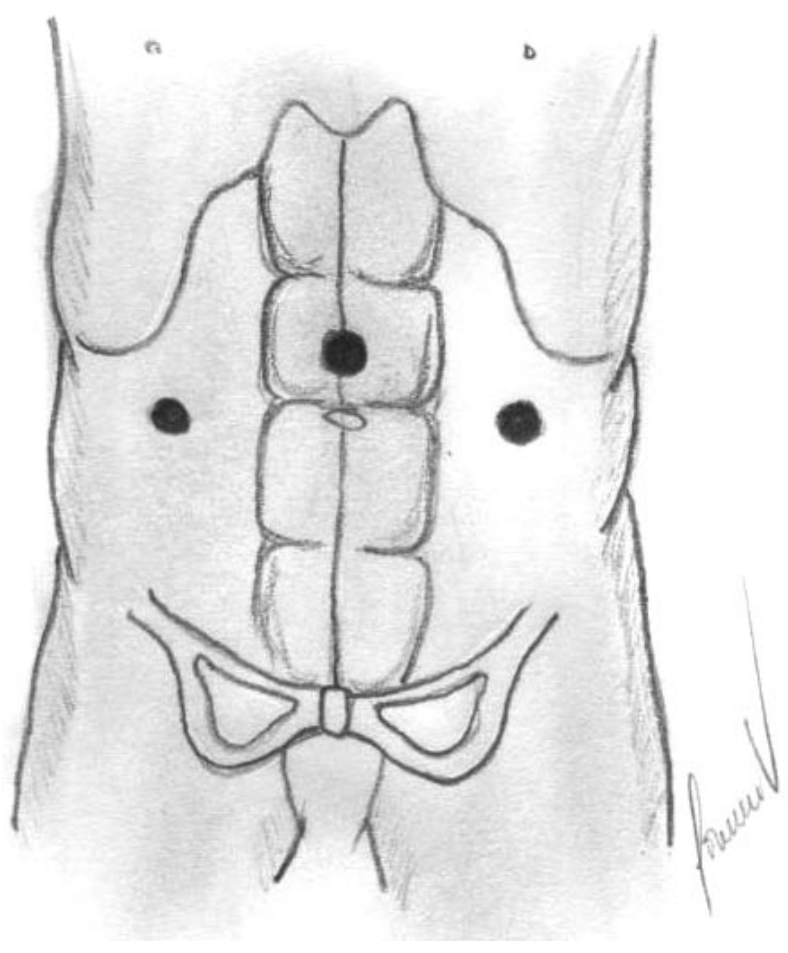

FIGURA 2. Colocación de los trócares: $10 \mathrm{~mm}$ a nivel umbilical para la óptica, y dos de $10 \mathrm{~mm}$ a nivel pararectal-paraumbilical para el resto del instrumental. 


\section{DISCUSIÓN}

La cistectomía parcial, es un procedimiento de preservación vesical que se basa en la resección quirúrgica de la pared vesical comprometida por la lesión. Este procedimiento se lleva a cabo desde el siglo XIX, pero adquirió mayor popularidad en la década de 1950 debido a su utilización habitual en pacientes con cáncer de vejiga (3). Posteriormente, el advenimiento de la resección transuretral de vejiga (RTUV), posibilitó el tratamiento conservador de algunos tumores, sin embargo, más tarde se observó que en muchos casos el carcinoma se encontraba más allá del epitelio de transición, invadiendo la porción muscular de la vejiga, asociándose a tasas de recurrencia local entre el 40 y el $78 \%$ (4).

Lo anterior significó que el tratamiento mínimamente invasivo y con preservación vesical se reserve sólo para pacientes muy seleccionados. En este sentido, las evidentes mejoras en las habilidades laparoscópicas de los urólogos y el desarrollo de nuevo instrumental en los últimos años han posibilitado el desarrollo de la cirugía reconstructiva laparoscópica del tracto urinario inferior. De esta manera, la cistectomía parcial laparoscópica entrega una serie de ventajas de la cirugía mínimamente invasiva sin dejar de lado los conceptos básicos de la cirugía a cielo abierto, permitiendo ampliar el espectro de pacientes favorecido por la cirugía mínimamente invasiva.

De acuerdo con lo anterior, estudios previos han demostrado que la cistectomía laparoscópica es factible y aporta claros beneficios al paciente, como lo son disminución de la morbilidad, regreso precoz del paciente a sus actividades diarias y un período más corto de la hospitalización (5). Mariano y Tefilli (6) presentaron recientemente una serie de 6 pacientes con cáncer invasor vesical en los que se realizó una cistectomía parcial laparoscópica mas linfadenectomía. El tiempo operatorio promedio fue de 205 min (rango 150 - 260) con un sangrado intraoperatorio promedio de $100 \mathrm{ml}$ (rango $100-300 \mathrm{ml}$ ), y una hospitalización promedio de 4 días. Desde el punto de vista oncológico, las biopsias de las piezas operatorias mostraron un caso con un estadío pT1G3, tres con estadío pT2aG2, un estadío pT2bG2 y un estadío pT3aG3. El tiempo de seguimiento promedio fue de 30 meses con un $80 \%$ de los casos libres de recurrencia. La única recurrencia, tanto local como sistémica se registro en el paciente con estadío pT3aG3, por lo que se indicó quimioterapia de salvataje. En el análisis global, sin embargo, nos parece que los resultados de esta serie son bastante alentadores, logrando la preservación vesical con un criterio oncológico.
Por otra parte Gerber y cols reportan el uso del $\mathrm{Nd}$ : YAG láser y laparoscopía simultánea en 5 pacientes con carcinoma de células transicionales de vejiga no invasor de alto grado. El abordaje laparoscópico permitió el desplazamiento de las asas intestinales lejos de la vejiga y la supervisión del uso del láser intravesical. Sin embargo, los resultados de este reporte mostraron una recurrencia de la enfermedad en 4 de los 5 pacientes en los siguientes 9 meses (7). A pesar de lo anterior, nos parece que el doble abordaje laparoscópico y endoscópico utilizado en nuestra serie y reportado por Gerber y cols. ofrece ventajas en la cistectomía parcial, ya que permite controlar el margen de resección de una manera objetiva.

En vista de lo anterior, en el caso especifico del cáncer vesical invasor, la cistectomía parcial debe ser reservada para un grupo de pacientes cuidadosamente seleccionados (8). Pacientes portadores de lesiones únicas con invasión muscular, ausencia de carcinoma in situ, sin antecedente de lesiones múltiples, localización diferente al trígono o uretra posterior y que permita garantizar un margen de seguridad oncológica de 1.5 a 2 centímetros serían buenos candidatos para la cistectomía parcial. En este tipo de lesiones los beneficios de la cistectomía parcial comprenden la posibilidad de una estadificación histopatológica completa del tumor y los ganglios linfáticos pelvianos y la preservación de la continencia, potencia y función vesical, traduciéndose en una mejor calidad de vida (9).

En cuanto a la sobrevida, las series clásicas de cistectomía parcial alcanzan cifras considerables de supervivencia a 5 años del $50 \%$ a $70 \%$ cuando se emplean estrictos criterios de selección $(8,10,11)$. Debe llamar la atención, sin embargo, que menos del $10 \%$ de los pacientes reportados en extensas series de cáncer vesical y sólo $5,8 \%$ a $19,9 \%$ de los pacientes con cáncer vesical invasor cumplen los criterios para la indicación de una cistectomía parcial (12). Es por eso que la selección adecuada del paciente es esencial para optimizar la tasa de supervivencia (13).

En nuestra serie, los casos de patología maligna por células transicionales y carcinoma de uraco fueron $2(22.2 \%)$ y se realizó linfadenectomía pélvica en cada caso tal como ha sido descrito en la literatura.

Como ya lo hemos señalado previamente, consideramos de vital importancia la selección adecuada del paciente. En este sentido, las contraindicaciones absolutas comprenden la presencia de un carcinoma in situ (CIS) o de tumores multifocales, mientras que las contraindicaciones relativas serían la presencia de 
tumores de alto grado, tumores localizados en el trígono o el cuello de la vejiga o tumores que requieran reimplantación ureteral posterior $(14,15)$.

Otras indicaciones potenciales para usar la técnica serían los tumores vesicales incluidos en divertículos y el adenocarcinoma del uraco que se origine en la cúpula vesical y se extienda hacia el ombligo a través del uraco $(2,8,10)$. Es fundamental señalar, que al igual que en la cirugía abierta, tanto en la carcinoma del uraco como en las lesiones adquiridas de este, está indicada la resección total de esta estructura, desde la cicatriz umbilical hasta el ápex vesical $(10,1)$. Sin embargo, algunos autores han descrito el tratamiento de las lesiones benignas del uraco, sin incluir la cistectomía parcial (17).

Además, este procedimiento es una opción quirúrgica para una diversidad de lesiones malignas menos frecuentes de la vejiga, como feocromocitomas (11), osteosarcoma primarios (18) y schwannoma vesical (19) así como lesiones benignas del tipo endometrioma $(8,12,20-22)$ y leiomioma vesical (17).

La cistectomía parcial laparoscópica no parece ser técnicamente difícil, sin embargo sugerimos y concordamos con la literatura en la importancia de la experiencia previa en cirugía pélvica urológica laparoscopica, así como en la necesidad de entrenamiento en sutura intracorpórea $(20,23,24)$, con la finalidad de obtener los mejores resultados. Es de suma importancia señalar que en los casos de lesiones malignas el seguimiento estricto es fundamental dada la real posibilidad de recurrencia y la eventual necesidad de terapias ayudantes.

\section{CONCLUSIONES}

La cistectomía parcial laparoscópica es un procedimiento quirúrgico factible y aplicable tanto para patología vesical benigna como maligna. La experiencia en cirugía reconstructiva urológica y el entrenamiento en sutura intracorpórea son fundamentales para realizar este procedimiento En los tumores vesicales de células transicionales y carcinomas del uraco, los criterios oncológicos deben prevalecer, lo que se logra con la selección adecuada de los pacientes. Si bien es cierto la cistectomía parcial aún cumple un rol limitado en el tratamiento del cáncer de vejiga en pacientes seleccionados, este procedimiento puede ser realizado por vía laparoscópica con resultados similares y baja morbilidad, cuando se compara con la cistectomía radical.

\section{BIBLIOGRAFÍA y LECTURAS RECOMENDADAS ( ${ }^{*}$ lectura de interés $y^{* *}$ lectura fundamental)}

1. THRASHER, J.B.; CRAWFORD, E.D.: "Current management of invasive and metastatic transitional cell carcinoma of the bladder". J. Urol., 149: 957, 1993.

**2. WADHWA, P.; KOLLA, S.B.; HEMAL, A.K.: "Laparoscopic en bloc partial cystectomy with bilateral pelvic lymphadenectomy for urachal adenocarcinoma”. Urology, 67: 837, 2006.

**3. LINDAHL, F.; JORGENSEN, P.; EGVAD, K.: "Partial cystectomy for transitional cell carcinoma of the bladder". Scand. J. Urol. Nephrol., 18: $125,1984$.

4. GIVEN, R.W.; WAJSMAN, Z.: "Bladder sparing treatments for muscle-invasive transitional cell carcinoma of the bladder". AUA Update Series, 16: 41, 1997.

5. CUMMINGS, K.B.; MASON, J.T.; CORREA, R.J. Jr. y cols.: "Segmental resection in the management of bladder carcinoma". J. Urol., 119: 56, 1978.

*6. MARIANO, M.B.; TEFILLI, M.V.: "Laparoscopic partial cystectomy in bladder cancer-initial experience”. Int. Braz. J. Urol., 30: 192, 2004.

*7. GERBER, G.S.; CHODAK, G.W.; RUKSTALIS, D.B.: "Combined laparoscopic and transurethral neodymium: yttriumaluminum-garnet laser treatment of invasive bladder cancer". Urology, 45: 230, 1995.

8. CHAPRON, C.; DUBUISSON, J.B.; JACOB, S. y cols.: "Laparoscopy and bladder endometriosis". Gynecol. Obstet. Fertil., 28: 232, 2000.

9. BURNETT, A.L.; EPSTEIN, J.I.; MARSHALL, F.F.: "Adenocarcinoma of the urinary bladder: Classification and management". Urology, 37: 315, 1991.

10. CADEDDU, J.A.; BOYLE, K.E.; FABRICIO, M.D. y cols.: "Laparoscopic management of urachal cysts in adulthood". J. Urol., 164: 1526, 2000.

11. KOZLOWSKI, P.M.; MIHM, F.; WINFIELD, H.N.: "Laparoscopic management of bladder pheochromocytoma". Urology, 57: 365, 2001.

**12. RICOS, T.J.V.; SOLSONA, N.E.: "Surgical treatment of urinary bladder cancer. Indications, technics, and general results". Arch. Esp. Urol., 52: 577, 1999.

**13. SWEENY, P.; KURSH, E.D.; RESNICK, M.I.: "Partial cystectomy". Urol. Clin. North Am., 19: 701, 1992.

14. BRANNAN, W.; OCHSNER, M.G.; FUSELIER, H.A. Jr. y cols.: "Partial cystectomy in the treatment of transitional cell carcinoma of the bladder". J. Urol., 119: 213, 1978. 
15. KANETI, J.: "Partial cystectomy in the management of bladder carcinoma". Eur. Urol., 12: 249, 1986.

16. NAVARRETE, S.; SANCHEZ-ISMAYEL, A.; SANCHEZ-SALAS, R.E. y cols.: "Treatment of urachal anomalies: A minimally invasive surgery technique". JSLS, 9: 422, 2005.

17. CHUNG, S.D.; TAI, H.C.; CHUEH, S.C.: "Laparoscopic partial cystectomy for a vesical leiomyoma". Int. Urogynecol. J. Pelvic Floor Dysfunct., 8: 223, 2006.

18. KATO, T.; KUBOTA, Y.: SAITOU, M. y cols.: "Osteosarcoma of the bladder successfully treated with partial cystectomy". J. Urol., 163: 548, 2000.

19. GEOL, H.; KIM, D.W.; KIM, T.H. y cols.: "Laparoscopic partial cystectomy for schwannoma of urinary bladder: case report". J. Endourol., 19: 303, 2005.

20. NEZHAT, C.H.; MALIK, S.; OSIAS, J. y cols.: "Laparoscopic management of 15 patients with infiltrating endometriosis of the bladder and a case of primary intravesical endometrioid adenosarcoma”. Fertil Steril, 78: 872, 2002.

21. SENER, A.; CHEW, B.H.; DUVDEVANI, M. y cols.: "Combined transurethral and laparoscopic partial cystectomy robot-assisted bladder repair for the treatment of bladder endometrioma". J. Minim. Invasive Gynecol., 13: 245, 2006.

*22. VITAGLIANO, G.; VILLETA, M.; CASTILLO, O.: "Laparoscopic partial cystectomy in the management of bladder endometriosis: Report of two cases". J. Endourol., 20: 1072, 2006.

23. ANDERSON, K.R.; CLAYMAN, R.V.: "Laparoscopic lower urinary tract reconstruction". World J. Urol., 18: 349, 2000.

24. NEZHAT, C.H.; SIEDMAN, D.S.; ROTTENBERG, H.: "Laparoscopic Management of Intentional and Unintentional Cystotomies". J. Am. Assoc. Gynecol. Laparosc., 3: 34, 1996. 